\title{
El control estricto de la glucemia disminuyó las complicaciones microvasculares en diabéticos de tipo 2
}

\begin{abstract}
Intensive blood-glucose control with sulphonylureas or insulin compared with conventional treatment and risk of complications in
\end{abstract} patients with type 2 diabetes (UKPDS 33). Committee ad hoc. Turner R., Holman R., Cull C. THE LANCET 1998; 352: 837-853.

\section{Objetivo}

Comparar los efectos del control estricto de la glucemia con sulfonilureas o insulina, con el tratamiento convencional en diabéticos tipo 2 (DBT2), sobre las complicaciones a largo plazo.

\section{Diseño}

Ensayo clínico aleatorizado con seguimiento promedio de 10 años. El reclutamiento se inició en 1977.

\section{Lugar}

23 hospitales del Reino Unido.

\section{Pacientes}

Pacientes con diagnóstico reciente de DBT2, $(n=4209), 58 \%$ hombres, edad promedio 54 años, sin trastornos metabólicos, renales, cardiovasculares agudos o retinopatía diabética que requiera fotocoagulación al momento de la inclusión.

\section{Intervención}

Los pacientes sin sobrepeso fueron aleatorizados a tratamiento intensivo(TI): meta de glucemia < a $110 \mathrm{mg}$, con sulfonilureas o insulina; y tratamiento convencional (TC): meta de glucemia < a $270 \mathrm{mg}$, con dieta. Si los controles, realizados cada tres meses, superaban el valor de glucemia requerido en cada grupo, los pacientes eran nuevamente aleatorizados a tratamiento con sulfonilureas, metformina o insulina, según tratamiento previo y criterios convencionales hasta alcanzar la meta correspondiente en cada grupo.

Los pacientes con sobrepeso fueron seleccionados para recibir metformina y los resultados de esta rama se reportaron en otro artículo ${ }^{1}$.

\section{Medición de resultados principales}

Fueron medidas 21 patologías; la mayoría relacionadas a la DBT2: Infarto de miocardio (IAM) fatal y no fatal, muerte súbita fatal, insuficiencia cardíaca, angina, accidente cerebrovascular (ACV) fatal y no fatal, muerte por enfermedad vascular periférica, amputación, muerte de causa renal, insuficiencia renal, fotocoagulación de retina, hemorragia vítrea, ceguera de un ojo, extracción de cataratas, muerte por hipo e hiperglucemia, accidente fatal, muerte por cáncer, muerte de cualquier otra causa y muerte de causa desconocida. Se realizó también un subanálisis de combinaciones de patologías en siete grupos: cualquier patología relacionada y muerte relacionada a la DBT2, todas las causas de mortalidad, IAM, ACV, amputación o muerte por enfermedad vascular periférica, y complicaciones microvasculares.

\section{Resultados principales}

Al cabo de 10 años la glucemia y la hemoglobina glicosilada fueron menores en el grupo TI que en el grupo control, sin bien ambos grupos aumentaron los valores a lo largo del seguimiento. El aumento de peso y la insulinemia fueron mayores en el grupo $\Pi I$ (promedio $2.9 \mathrm{Kg}$ $p=<0.001$ para el primero) que en el grupo TC.

\begin{tabular}{|c|c|c|c|c|c|}
\hline Evento & $\begin{array}{l}\text { Incidencia } \\
\text { por } 1000 \\
\text { pacientes/ } \\
\text { año } \\
\text { Grupo TC }\end{array}$ & $\begin{array}{l}\text { Incidencia } \\
\text { por } 1000 \\
\text { pacientes/ } \\
\text { año } \\
\text { Grupo } \pi \\
\end{array}$ & $\mathrm{RRA}^{*}$ & $\begin{array}{l}\mathrm{RR}^{*} \\
\text { (IC 95\%) }\end{array}$ & $\begin{array}{lc}\text { NNT* } \\
\text { (IC 95\%) }\end{array}$ \\
\hline $\begin{array}{l}\text { Patología relacionada } \\
\text { a DBT }\end{array}$ & $46 \%$ & $40.9 \%$ & $5 \%$ & $0.88(0.79-0.99)$ & $\begin{array}{rc}0.029 & 20 \\
(10-200)\end{array}$ \\
\hline Muerte relacionàda a DBT & $11.5 \%$ & $10.4 \%$ & $1 \%$ & $0.90(0.73-1.11)$ & NS \\
\hline Muerte por cualquier causa & $18.9 \%$ & $17.9 \%$ & $1 \%$ & $0.94(0.80-1.10)$ & NS \\
\hline Complicaciones microvasc & c. $11.4 \%$ & $8.6 \%$ & $2 \%$ & $0.75(0.60-0.93)$ & $\begin{array}{r}<0.00150 \\
(22-125)\end{array}$ \\
\hline IAM & $17.4 \%$ & $14.7 \%$ & $2.5 \%$ & $0.84(0.71-1)$ & 0.05240 \\
\hline
\end{tabular}

Los NNT se colocan sólo en los que el resultado fue estadísticamente significativo o marginalmente significativo.

En el subanálisis por grupos de tratamientos se observó mayor beneficio en los pacientes que recibieron insulina y glibenclamida.

La mayor reducción de riesgo fue dada por disminución del $25 \%$ de complicaciones en la microangiopatía en su mayor parte debido a la reducción de necesidad de fotoçoagulación RR 0.71 (IC 0.53 a 0.96 , $\mathrm{p}=0.0031$ ).

Los épisodios de hipoglucemia fueron mayores en el grupo de TI ( $1.8 \%$ anual con insulina, $1.4 \%$ con glibenclamida, $1 \%$ con clorpropamida) que en el grupo TC $(0,7 \%$ anual $)$.

\section{Conclusiones}

El tratamiento intensivo de la diabetes tipo 2 con glibenclamida o insulina es beneficioso para reducir las complicaciones microvasculares, sobre todo la retinopatía. El tratamiento intensivo aumenta el riesgo de hipoglucemia pero no se observaron complicaciones fatales por la misma.

\section{COMENTARIO}

Este trabajo generó gran expectativa porque desde la publicación del DCCT en $1993^{2}$, que demostraba la eficacia en la prevención de enfermedad microvascular en diabéticos tipo 1 con múltiples inyecciones diarias de insulina, se debatió acerca de la generalización de sus resultados y la utilidad del tratamiento intensivo en pacientes con DBT2. Si bien algunos ensayos clínicos controlados aleatorizados y bastante evidencia epidemiológica sugerían un efecto beneficioso ${ }^{3}$, éste quedaba aún sin demostrar. Este es el primer trabajo aleatorizado en DBT2 que certifica que el tratamiento más estricto es mejor en población occidental. Ahora bien, para trasladar sus resultados a la práctica existen algunos inconvenientes: 1) la población con TC tenía glucemias más altas que las que toleramos habitualmente en el consultorio (meta de glucemia $<270 \mathrm{mg} \%$ ) con lo cual el beneficio en nuestra práctica diaria se vería diluido, y el beneficio hallado en el estudio se acrecentaría;

\section{Dr. Pablo Tesolin}

Unidad de Medicina Familiar y Preventiva. Hospital Italiano de Buenos Aires.

\section{Dra. Marcela Botargues}

Unidad de Medicina Familiar y Preventiva. Hospital Italiano de Buenos Aires.

\section{Referencias}

1. Effect of inintensive blood-glucose control with metformin on complications in overwright patients wth type 2 diabetes. The Lancet 1998: $352: 854-865$.

2. The DCCT Research Group. The effect of intensive tratment of Diabetes on the development and porgresion of long term complications in insulin dependent diabetes mellitus. $\mathrm{N}$ Eng $\mathrm{J}$ Med.1993; 329:997-86

3. Intensive insulin therapy prevents the progression in Japanise patients with non insulin dependent diabetes mellitus:a ramdomized prospective 6 -year study. Diabetes res. Clin. Pract 1995:28: 103-17. Ohkubo Y, Kishikawa II, Araki E, et at.

2) de los 21 puntos finales simples sólo hubo diferencia significativa en la reducción de fotocoagulación retinal, y no en los 20 restantes; 3) de los 7 puntos finales combinados se observaron beneficios significativos del tratamiento intensivo en tres de ellos: patologías relacionadas a DBT2, IAM, y complicaciones microvasculares. Si bien los autores mencionan una reducción de la patología y muerte relacionada a la diabetes e infarto, ésta no alcanzó significación estadística.

Entonces, ¿qué nos agrega este estudio a nuestra práctica? : es un aval científico para que la meta de glucemia en DBT2 sea más estricta. Lo propuesto por los paneles de expertos es glucemia en ayunas $<140$ y postprandial $<180^{4}$, en este trabajo la meta era menor de $110 \mathrm{mg} \%$. Probablemente la causa más frecuente de in cumplimiento de las metas sea que los pacientes no suelen seguir las recomendaciones médicas. El primer planteo para nuestra práctica sería convencernos de que la meta es la normalización de la glucemia y en pacientes motivados escalonar el tratamiento en esa dirección. En pacientes menos adherentes al tratamiento, deberíamos ser más laxos para evitar las complicaciones del mismo (hipoglucemia). 